\title{
Two-Step Time of Arrival Estimation for Pulse-Based Ultra-Wideband Systems
}

\author{
Sinan Gezici, ${ }^{1}$ Zafer Sahinoglu, ${ }^{2}$ Andreas F. Molisch, ${ }^{2}$ Hisashi Kobayashi, ${ }^{3}$ and H. Vincent Poor ${ }^{3}$ \\ ${ }^{1}$ Department of Electrical and Electronics Engineering, Bilkent University, Bilkent, Ankara 06800, Turkey \\ ${ }^{2}$ Mitsubishi Electric Research Labs, 201 Broadway, Cambridge, MA 02139, USA \\ ${ }^{3}$ Department of Electrical Engineering, Princeton University, Princeton, NJ 08544, USA \\ Correspondence should be addressed to Sinan Gezici, gezici@ieee.org
}

Received 12 November 2007; Revised 12 March 2008; Accepted 14 April 2008

Recommended by Davide Dardari

\begin{abstract}
In cooperative localization systems, wireless nodes need to exchange accurate position-related information such as time-of-arrival (TOA) and angle-of-arrival (AOA), in order to obtain accurate location information. One alternative for providing accurate position-related information is to use ultra-wideband (UWB) signals. The high time resolution of UWB signals presents a potential for very accurate positioning based on TOA estimation. However, it is challenging to realize very accurate positioning systems in practical scenarios, due to both complexity/cost constraints and adverse channel conditions such as multipath propagation. In this paper, a two-step TOA estimation algorithm is proposed for UWB systems in order to provide accurate TOA estimation under practical constraints. In order to speed up the estimation process, the first step estimates a coarse TOA of the received signal based on received signal energy. Then, in the second step, the arrival time of the first signal path is estimated by considering a hypothesis testing approach. The proposed scheme uses low-rate correlation outputs and is able to perform accurate TOA estimation in reasonable time intervals. The simulation results are presented to analyze the performance of the estimator.
\end{abstract}

Copyright (c) 2008 Sinan Gezici et al. This is an open access article distributed under the Creative Commons Attribution License, which permits unrestricted use, distribution, and reproduction in any medium, provided the original work is properly cited.

\section{INTRODUCTION}

Recently, communications, positioning, and imaging systems that employ ultra-wideband (UWB) signals have drawn considerable attention [1-5]. Commonly, a UWB signal is defined to be one that possesses an absolute bandwidth of at least $500 \mathrm{MHz}$ or a relative bandwidth larger than $20 \%$. The main feature of a UWB signal is that it can coexist with incumbent systems in the same frequency range due to its large spreading factor and low power spectral density. UWB technology holds great promise for a variety of applications such as short-range, high-speed data transmission and precise position estimation $[2,6]$.

A common technique to implement a UWB communications system is to transmit very short-duration pulses with a low duty cycle [7-11]. Such a system, called impulse radio (IR), sends a train of pulses per information symbol and usually employs pulse position modulation (PPM) or binary-phase shift keying (BPSK) depending on the positions or the polarities of the pulses, respectively. In order to prevent catastrophic collisions among pulses of different users and thus provide robustness against multiple access interference (MAI), each information symbol is represented by a sequence of pulses; the positions of the pulses within that sequence are determined by a pseudo-random time hopping (TH) sequence specific to each user [7].

In addition to communications systems, UWB signals are also well suited for applications that require accurate position information such as inventory control, search and rescue, and security $[3,12]$. They are also useful in the context of cooperative localization systems, since exchange of accurate position-related information is very important for efficient cooperation. In the presence of inaccurate position-related information, cooperation could be harmful by reducing the localization accuracy. Therefore, high TOA estimation accuracy of UWB signals is very desirable in cooperative localization systems. Due to their penetration capability and high time resolution, UWB signals can facilitate very precise positioning based on time-of-arrival (TOA) estimation, as suggested by the Cramer-Rao lower bound (CRLB) [3]. 
However, in practical systems, the challenge is to perform precise TOA estimation in a reasonable time interval under complexity/cost constraints [13].

Maximum likelihood (ML) approaches to TOA estimation of UWB signals can get quite close to the theoretical limits for high signal-to-noise ratios (SNRs) [14, 15]. However, they generally require joint optimization over a large number of unknown parameters (channel coefficients and delays for multipath components). Hence, they have prohibitive complexity for practical applications. In [16], a generalized maximum likelihood (GML) estimation principle is employed to obtain iterative solutions after some simplifications of the ML approach. However, this approach still requires very high sampling rates, which is not suitable for low-power and low-cost applications.

On the other hand, the conventional correlation-based TOA estimation algorithms are both suboptimal and require exhaustive search among thousands of bins, which results in very slow TOA estimation $[17,18]$. In order to speed up the process, different search strategies such as random search or bit reversal search are proposed in [19]. However, TOA estimation time can still be quite high in certain scenarios. In addition to the correlation-based TOA estimation, TOA estimation based on energy detection provides a lowcomplexity alternative, but this commonly comes at the price of reduced accuracy $[20,21]$.

In the presence of multipath propagation, the first incoming signal path, the delay of which determines the TOA, may not be the strongest multipath component. Therefore, instead of peak selection algorithms, first path detection algorithms are commonly employed for UWB TOA estimation [16, 21-25]. A common technique for first path detection is to determine the first signal component that is stronger than a specific threshold [25]. Alternatively, the delay of the first path can be estimated based on the signal path that has the minimum delay among a subset of signal paths that are stronger than a certain threshold [24]. Although TOA estimation gets more robust against the effects of multipath propagation in both cases, TOA estimation can still take a long time. Finally, a lowcomplexity timing offset estimation technique, called timing with dirty templates (TDT), is proposed in [23, 26-28], which employs "dirty templates" in order to obtain timing information based on symbol-rate samples. Although this algorithm provides timing information at low complexity and in short time intervals, the TOA estimate obtained from the algorithm has an ambiguity equal to the extent of the noise-only region between consecutive symbols.

One of the most challenging issues in UWB TOA estimation is to obtain a reliable estimate in a reasonable time interval under a constraint on sampling rate. In order to have a low-power and low-complexity receiver, one should assume low sampling rates at the output of the correlators. However, when low-rate samples are employed, the TOA estimation can take a very long time. Therefore, we propose a two-step TOA estimation algorithm that can perform TOA estimation from low-rate samples (typically on the order of hundreds times slower sampling rate than chip-rate sampling) in a reasonable time interval. In order to speed up the estimation process, the first step estimates the coarse TOA of the received signal based on received signal energy. After the first step, the uncertainty region for TOA is reduced significantly. Then, in the second step, the arrival time of the first signal path is estimated based on low-rate correlation outputs by considering a hypothesis testing approach. In other words, the second step provides a fine TOA estimate by using a statistical change detection approach. In addition, the proposed algorithm can operate without any thresholding operation, which increases its practicality.

The remainder of the paper is organized as follows. Section 2 describes the transmitted and received signal models in a frequency-selective environment. The two-step TOA estimation algorithm is considered in Section 3, where the algorithm is described in detail, and probability of detection analysis is presented. Then, simulation results and numerical studies are presented in Section 4, and concluding remarks are made in Section 5.

\section{SIGNAL MODEL}

Consider a TH-IR system which transmits the following signal:

$$
s_{\mathrm{tx}}(t)=\sqrt{E} \sum_{j=-\infty}^{\infty} a_{j} b_{\left\lfloor j / N_{\mathrm{f}}\right\rfloor} w_{\mathrm{tx}}\left(t-j T_{\mathrm{f}}-c_{j} T_{\mathrm{c}}\right)
$$

where $w_{\mathrm{tx}}(t)$ is the transmitted UWB pulse with duration $T_{\mathrm{c}}$; $E$ is the transmitted pulse energy; $T_{\mathrm{f}}$ is the "frame" interval; and $b_{\left\lfloor j / N_{f}\right\rfloor} \in\{+1,-1\}$ is the binary information symbol. In order to smooth the power spectrum of the transmitted signal and allow the channel to be shared by many users without causing catastrophic collisions, a TH sequence $c_{j} \in$ $\left\{0,1, \ldots, N_{c}-1\right\}$ is assigned to each user, where $N_{c}$ is the number of chips per frame interval, that is, $N_{c}=T_{\mathrm{f}} / T_{\mathrm{c}}$. Additionally, random polarity codes, $a_{j}$ 's, can be employed, which are binary random variables taking on the values \pm 1 with equal probability, and are known to the receiver. Use of random polarity codes helps reduce the spectral lines in the power spectral density of the transmitted signal $[29,30]$ and mitigate the effects of MAI $[31,32]$.

It can be shown that the signal model in (1) also covers the signal structures employed in the preambles of IEEE 802.15.4a systems [2, 33].

The transmitted signal in (1) passes through a channel with channel impulse response $h(t)$, which is modeled as a tapped-delay-line channel with multipath resolution $T_{\mathrm{c}}$ as follows [34-36]:

$$
h(t)=\sum_{l=1}^{L} \alpha_{l} \delta\left(t-(l-1) T_{\mathrm{c}}-\tau_{\mathrm{TOA}}\right),
$$

where $\alpha_{l}$ is the channel coefficient for the $l$ th path; $L$ is the number of multipath components; and $\tau_{\mathrm{TOA}}$ is the TOA of the incoming signal. Since the main purpose is to estimate TOA with a chip-level uncertainty, the equivalent channel model with resolution $T_{\mathrm{c}}$ is employed. 
From (1) and (2), and including the effects of the antennas, the received signal can be expressed as

$$
r(t)=\sum_{l=1}^{L} \sqrt{E} \alpha_{l} s_{\mathrm{rX}}\left(t-(l-1) T_{\mathrm{c}}-\tau_{\mathrm{TOA}}\right)+n(t),
$$

where $n(t)$ is zero-mean white Gaussian noise with spectral density $\sigma^{2}$; and $s_{\mathrm{rx}}(t)$ is given by

$$
s_{\mathrm{rx}}(t)=\sum_{j=-\infty}^{\infty} a_{j} b_{\left\lfloor j / N_{\mathrm{f}}\right\rfloor} w_{\mathrm{rx}}\left(t-j T_{\mathrm{f}}-c_{j} T_{\mathrm{c}}\right),
$$

with $w_{\mathrm{rx}}(t)$ denoting the received UWB pulse with unit energy.

Since TOA estimation is commonly performed at the preamble section of a packet [33], we assume a data aided TOA estimation scheme and consider a training sequence of $b_{j}=1 \forall j$. Then, $s_{\mathrm{rx}}(t)$ in (4) can be expressed as

$$
s_{\mathrm{rX}}(t)=\sum_{j=-\infty}^{\infty} a_{j} w_{\mathrm{rx}}\left(t-j T_{\mathrm{f}}-c_{j} T_{\mathrm{c}}\right) .
$$

It is assumed, for simplicity, that the signal always arrives in one frame duration $\left(\tau_{\mathrm{TOA}}<T_{\mathrm{f}}\right)$, and there is no interframe interference (IFI), that is, $T_{\mathrm{f}} \geq\left(L+c_{\max }\right) T_{\mathrm{c}}$ (equivalently, $N_{c} \geq L+c_{\max }$ ), where $c_{\max }$ is the maximum value of the TH sequence. Note that the assumption of $\tau_{\mathrm{TOA}}<T_{\mathrm{f}}$ does not restrict the validity of the algorithm. In fact, it is enough to have $\tau_{\mathrm{TOA}}<T_{\mathrm{s}}$, where $T_{\mathrm{s}}$ is the symbol interval, for the algorithm to work when the frame interval is large enough and predetermined $\mathrm{TH}$ codes are employed. (In fact, in IEEE 802.15.4a systems, no TH codes are used in the preamble section; hence, it is easy to extend the results to the $\tau_{\mathrm{TOA}}>T_{\mathrm{f}}$ case for those scenarios [2].) Moreover, even if $\tau_{\mathrm{TOA}} \geq T_{\mathrm{s}}$, an initial energy detection can be used to determine the arrival time within a symbol uncertainty before running the proposed algorithm. Finally, since a single-user scenario is considered, $c_{j}=0 \forall j$ can be assumed without loss of generality.

\section{TWO-STEP TOA ESTIMATION ALGORITHM}

A TOA estimation algorithm provides an estimate for the delay of an incoming signal, which is commonly obtained in multiple steps, as shown in Figure 1. First, frame acquisition is achieved in order to confine the TOA into an uncertainty region of one frame interval (see [37]). Then, the TOA is estimated with a chip-level uncertainty by a TOA estimation algorithm, which is shown in the dashed box in Figure 1. Then, the tracking unit provides subchip resolution by employing a delay lock loop (DLL), which yields the final TOA estimate [38-40]. The focus of this paper is on the twostep TOA estimation algorithm shown in Figure 1.

In order to perform fast TOA estimation, the first step of the proposed two-step TOA estimation algorithm obtains a coarse TOA of the received signal based on received signal energy. Then, in the second step, the arrival time of the first signal path is estimated by considering a hypothesis testing approach.

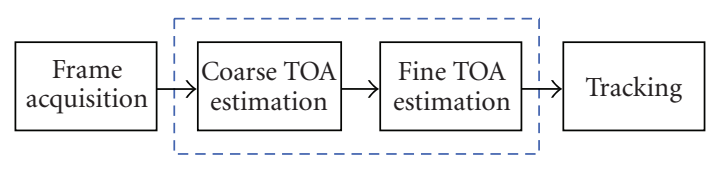

FIGURE 1: Block diagram for TOA estimation. The algorithm in this paper focuses on the blocks in the dashed box.

First, the TOA $\tau_{\mathrm{TOA}}$ in (3) is expressed as

$$
\tau_{\mathrm{TOA}}=k T_{\mathrm{c}}=k_{\mathrm{b}} T_{\mathrm{b}}+k_{c} T_{\mathrm{c}},
$$

where $k \in\left[0, N_{c}-1\right]$ is the TOA in terms of the chip interval $T_{\mathrm{c}} ; T_{\mathrm{b}}$ is the block interval consisting of $B$ chips $\left(T_{\mathrm{b}}=B T_{\mathrm{c}}\right)$; and $k_{b} \in\left[0, N_{c} / B-1\right]$ and $k_{c} \in[0, B-1]$ are the integers that determine, respectively, in which block and chip the first signal path arrives. Note that $N_{c} / B$ represents the number of blocks, which is denoted by $N_{\mathrm{b}}$ in the sequel.

The two-step TOA algorithm first estimates the block in which the first signal path exists. Then, it estimates the chip position in which the first path resides. In other words, it can be summarized as follows:

(i) estimation of $k_{b}$ from received signal strength (RSS) measurements;

(ii) estimation of $k_{c}$ (equivalently, $k$ ) from low-rate correlation outputs using a hypothesis testing approach.

Note that the number of blocks $N_{\mathrm{b}}$ (or the block length $T_{\mathrm{b}}$ ) is an important design parameter. Selection of a smaller block decreases the amount of time for TOA estimation in the second step, since a smaller uncertainty region is searched. On the other hand, smaller block sizes can result in more errors in the first step since noise becomes more effective. The optimal block size is affected by the SNR and the channel characteristics.

\subsection{First step: coarse TOA estimation based on RSS measurements}

In the first step, the aim is to detect the coarse arrival time of the signal in the frame interval. Assume, without loss of generality, that the frame time $T_{\mathrm{f}}$ is an integer multiple of $T_{\mathrm{b}}$, the block size of the algorithm, that is, $T_{\mathrm{f}}=N_{\mathrm{b}} T_{\mathrm{b}}$.

In order to have reliable decision variables in this step, energy is combined from $N_{1}$ different frames of the incoming signal for each block. Hence, the decision variables are expressed as

$$
Y_{i}=\sum_{j=0}^{N_{1}-1} Y_{i, j}
$$

for $i=0, \ldots, N_{\mathrm{b}}-1$, where

$$
Y_{i, j}=\int_{j T_{\mathrm{f}}+i T_{\mathrm{b}}}^{j T_{\mathrm{f}}+(i+1) T_{\mathrm{b}}}|r(t)|^{2} d t .
$$

Then, $k_{b}$ in (6) is estimated as

$$
\hat{k}_{b}=\arg \max _{0 \leq i \leq N_{\mathrm{b}}-1} Y_{i} \text {. }
$$


In other words, the block with the largest signal energy is selected.

The parameters of this step that should be selected appropriately for accurate TOA estimation are the block size $T_{\mathrm{b}}\left(N_{\mathrm{b}}\right)$ and the number of frames $N_{1}$, from which energy is collected. In Section 3.4, the probability of selecting the correct block will be quantified.

\subsection{Second step: fine TOA estimation based on low-rate correlation outputs}

After determining the coarse arrival time in the first step, the second step tries to estimate $k_{c}$ in (6). Ideally, $k_{c} \in$ $[0, B-1]$ needs to be searched for TOA estimation, which corresponds to searching $k \in\left[\hat{k}_{b} B,\left(\hat{k}_{b}+1\right) B-1\right]$ with $\hat{k}_{b}$ denoting the block index estimate in (9). However, in some cases, the first signal path can reside in one of the blocks prior to the strongest one due to multipath effects. Therefore, instead of searching a single block, $k \in\left[\hat{k}_{b} B-\right.$ $\left.M_{1},\left(\hat{k}_{b}+1\right) B-1\right]$, with $M_{1} \geq 0$, can be searched for the TOA in order to increase the probability of detection of the first path. In other words, in addition to the block with the largest signal energy, an additional backwards search over $M_{1}$ chips can be performed. For notational simplicity, let $\mathcal{U}=\left\{n_{\mathrm{s}}, n_{\mathrm{s}}+1, \ldots, n_{\mathrm{e}}\right\}$ denote the uncertainty region, where $n_{\mathrm{s}}=\hat{k}_{b} B-M_{1}$ and $n_{\mathrm{e}}=\left(\hat{k}_{b}+1\right) B-1$ are the start and end points.

In order to estimate the TOA with chip-level resolution, correlations of the received signal with shifted versions of a template signal are considered. For delay $i T_{c}$, the following correlation output is obtained:

$$
z_{i}=\int_{i T_{\mathrm{c}}}^{i T_{\mathrm{c}}+N_{2} T_{\mathrm{f}}} r(t) s_{\mathrm{temp}}\left(t-i T_{\mathrm{c}}\right) d t,
$$

where $N_{2}$ is the number of frames over which the correlation output is obtained, and $s_{\text {temp }}(t)$ is the template signal given by

$$
s_{\text {temp }}(t)=\sum_{j=0}^{N_{2}-1} a_{j} w_{\mathrm{rx}}\left(t-j T_{\mathrm{f}}\right) .
$$

Note that in practical systems, the received pulse shape may not be known exactly, since the transmitted pulse can be distorted by the channel. In those cases, if the system employs $w_{\mathrm{tx}}(t)$ instead of $w_{\mathrm{rx}}(t)$ to construct the template signal in (11), the system performance can degrade. In some cases, that degradation may not be very significant [41]. For other cases, template design techniques should be considered in order to maintain a reasonable performance level $[41,42]$.

From the correlation outputs for different delays, the aim is to determine the chip in which the first signal path has arrived. By appropriate choice of the block interval $T_{\mathrm{b}}$ and $M_{\mathrm{l}}$, and considering a large number of multipath components in the received signal, which is typical for indoor UWB systems, it can be assumed that the block starts with a number of chips with noise-only components and the remaining ones with signal-plus-noise components, as

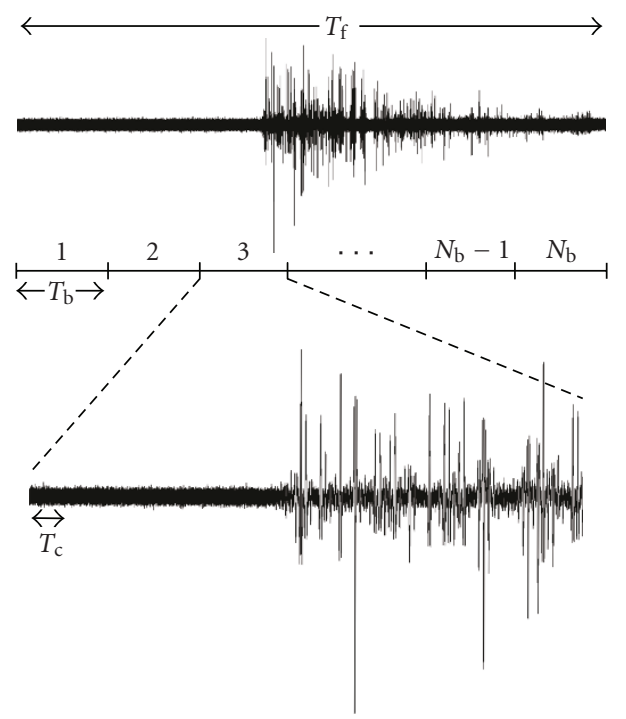

FIgURE 2: Illustration of the two-step TOA estimation algorithm. The signal on the top is the received signal in one frame. The first step checks the signal energy in $N_{\mathrm{b}}$ blocks and chooses the one with the highest energy (although one frame is shown in the figure, energy from different frames can be collected for reliable decisions). Assuming that the third block has the highest energy, the second step focuses on this block (or an extension of that) to estimate the TOA. The zoomed version of the signal in the third block is shown on the bottom.

shown in Figure 2. Assuming that the statistics of the signal paths do not change significantly in the uncertainty region $\mathcal{U}$, the different hypotheses can be expressed approximately as follows:

$$
\begin{gathered}
\mathscr{H}_{0}: z_{i}=\eta_{i}, \quad i=n_{\mathrm{s}}, \ldots, n_{\mathrm{f}}, \\
\mathcal{H}_{k}: z_{i}=\eta_{i}, \quad i=n_{\mathrm{s}}, \ldots, k-1, \\
z_{i}=N_{2} \sqrt{E} \alpha_{i-k+1}+\eta_{i}, \quad i=k, \ldots, n_{\mathrm{f}},
\end{gathered}
$$

for $k \in \mathcal{U}$, where $\mathscr{H}_{0}$ is the hypothesis that all the samples are noise samples; $\mathscr{H}_{k}$ is the hypothesis that the signal starts at the $k$ th output; $\eta_{i}$ 's denote the independent and identically distributed (i.i.d.) Gaussian output noise; $\mathcal{N}\left(0, \sigma_{n}^{2}\right)$ with $\sigma_{n}^{2}=N_{2} \sigma^{2}, \alpha_{1}, \ldots, \alpha_{n_{\mathrm{f}}-k+1}$ are independent channel coefficients, assuming $n_{\mathrm{f}}-n_{\mathrm{s}}+1 \leq L$, and $n_{\mathrm{f}}=$ $n_{\mathrm{e}}+M_{2}$ with $M_{2}$ being the number of additional correlation outputs that are considered out of the uncertainty region in order to have reliable estimates of the unknown parameters related to the channel coefficients.

Due to very time high resolution of UWB signals, it is appropriate to model the channel coefficients approximately as

$$
\begin{aligned}
& \alpha_{1}=d_{1}\left|\alpha_{1}\right|, \\
& \alpha_{l}=\left\{\begin{array}{ll}
d_{l}\left|\alpha_{l}\right|, & p, \\
0, & 1-p,
\end{array} \quad l=2, \ldots, n_{\mathrm{f}}-n_{\mathrm{s}}+1,\right.
\end{aligned}
$$

where $p$ is the probability that a channel tap arrives in a given chip; $d_{l}$ is the sign of $\alpha_{l}$, which is \pm 1 with equal probability; 
and $\left|\alpha_{l}\right|$ is the amplitude of $\alpha_{l}$, which is modeled as a Nakagami- $m$ distributed random variable with parameter $\Omega$, that is [43],

$$
p(\alpha)=\frac{2}{\Gamma(m)}\left(\frac{m}{\Omega}\right)^{m} \alpha^{2 m-1} e^{-m \alpha^{2} / \Omega},
$$

for $\alpha \geq 0, m \geq 0.5$, and $\Omega \geq 0$, where $\Gamma(\cdot)$ is the Gamma function [44].

From the formulation in (12), it is observed that the TOA estimation problem can be considered as a change detection problem [45]. Let $\boldsymbol{\theta}$ denote the unknown parameters of the distribution of $\alpha$, that is, $\boldsymbol{\theta}=[p m \Omega]$. Then, the loglikelihood ratio (LLR) is given by

$$
S_{k}^{n_{\mathrm{f}}}(\boldsymbol{\theta})=\sum_{i=k}^{n_{\mathrm{f}}} \log \frac{p_{\boldsymbol{\theta}}\left(z_{i} \mid \mathscr{H}_{k}\right)}{p\left(z_{i} \mid \mathscr{H}_{0}\right)}
$$

where $p_{\boldsymbol{\theta}}\left(z_{i} \mid \mathscr{H}_{k}\right)$ denotes the probability density function (p.d.f.) of the correlation output under hypothesis $\mathscr{H}_{k}$ and with unknown parameters given by $\boldsymbol{\theta}$, and $p\left(z_{i} \mid \mathscr{H}_{0}\right)$ denotes the p.d.f. of the correlation output under hypothesis $\mathscr{H}_{0}$.

Since $\boldsymbol{\theta}$ is unknown, its ML estimate can be obtained first for a given hypothesis $\mathscr{H}_{k}$ and then that estimate can be used in the LLR expression. In other words, the generalized LLR approach $[45,46]$ can be taken, where the TOA estimate is expressed as

$$
\hat{k}=\arg \max _{k \in \mathcal{U}} S_{k}^{n_{\mathrm{f}}}\left(\hat{\boldsymbol{\theta}}_{\mathrm{ML}}(k)\right)
$$

with

$$
\widehat{\boldsymbol{\theta}}_{\mathrm{ML}}(k)=\arg \sup _{\boldsymbol{\theta}} S_{k}^{n_{\mathrm{f}}}(\boldsymbol{\theta})
$$

However, the ML estimate is usually very complex to calculate. Therefore, simpler estimators such as the method of moments (MM) estimator can be employed to obtain those parameters. The $n$th moment of a random variable $X$ having Nakagami- $m$ distribution with parameter $\Omega$ is given by

$$
E\left\{X^{n}\right\}=\frac{\Gamma(m+n / 2)}{\Gamma(m)}\left(\frac{\Omega}{m}\right)^{n / 2}
$$

Then, from the correlator outputs $\left\{z_{i}\right\}_{i=k+1}^{n_{\mathrm{f}}}$, the MM estimates for the unknown parameters can be obtained after some manipulation as

$$
p_{\mathrm{MM}}=\frac{\gamma_{1} \gamma_{2}}{2 \gamma_{2}^{2}-\gamma_{3}}, \quad m_{\mathrm{MM}}=\frac{2 \gamma_{2}^{2}-\gamma_{3}}{\gamma_{3}-\gamma_{2}^{2}}, \quad \Omega_{\mathrm{MM}}=\frac{2 \gamma_{2}^{2}-\gamma_{3}}{\gamma_{2}},
$$

where

$$
\begin{aligned}
& \gamma_{1} \triangleq \frac{1}{E N_{2}^{2}}\left(\mu_{2}-\sigma_{n}^{2}\right), \\
& \gamma_{2} \triangleq \frac{1}{E^{2} N_{2}^{4}}\left(\frac{\mu_{4}-3 \sigma_{n}^{4}}{\gamma_{1}}-6 E N_{2}^{2} \sigma_{n}^{2}\right), \\
& \gamma_{3} \triangleq \frac{1}{E^{3} N_{2}^{6}}\left(\frac{\mu_{6}-15 \sigma_{n}^{6}}{\gamma_{1}}-15 E^{2} N_{2}^{4} \gamma_{2} \sigma_{n}^{2}-45 E N_{2}^{2} \sigma_{n}^{4}\right),
\end{aligned}
$$

with $\mu_{j}$ denoting the $j$ th sample moment given by

$$
\mu_{j}=\frac{1}{n_{\mathrm{f}}-k} \sum_{i=k+1}^{n_{\mathrm{f}}} z_{i}^{j} .
$$

Then, the index of the chip having the first signal path can be obtained as

$$
\widehat{k}=\arg \max _{k \in \mathcal{U}} S_{k}^{n_{\mathrm{f}}}\left(\widehat{\boldsymbol{\theta}}_{\mathrm{MM}}(k)\right),
$$

where $\boldsymbol{\theta}_{\mathrm{MM}}(k)=\left[p_{\mathrm{MM}} m_{\mathrm{MM}} \Omega_{\mathrm{MM}}\right]$ is the MM estimate for the unknown parameters. Note that the dependence of $p_{\mathrm{MM}}, m_{\mathrm{MM}}$, and $\Omega_{\mathrm{MM}}$ on the change position $k$ is not shown explicitly for notational simplicity.

Let $p_{1}(z)$ and $p_{2}(z)$, respectively, denote the distributions of $\eta$ and $N_{2} \sqrt{E} d|\alpha|+\eta$. Then, the generalized LLR for the $k$ th hypothesis can be obtained as

$$
S_{k}^{n_{\mathrm{f}}}(\hat{\boldsymbol{\theta}})=\log \frac{p_{2}\left(z_{k}\right)}{p_{1}\left(z_{k}\right)}+\sum_{i=k+1}^{n_{\mathrm{f}}} \log \frac{p p_{2}\left(z_{i}\right)+(1-p) p_{1}\left(z_{i}\right)}{p_{1}\left(z_{i}\right)}
$$

where

$$
\begin{aligned}
& p_{1}(z)=\frac{1}{\sqrt{2 \pi} \sigma_{n}} e^{-z^{2} / 2 \sigma_{n}^{2}} \\
& p_{2}(z)=\frac{\nu_{1}}{\sqrt{2 \pi} \sigma_{n}} e^{-z^{2} / 2 \sigma_{n}^{2}} \Phi\left(m, \frac{1}{2} ; \frac{z^{2}}{\nu_{2}}\right)
\end{aligned}
$$

with

$$
\begin{aligned}
& \nu_{1} \triangleq \frac{2 \sqrt{\pi} \Gamma(2 m)}{\Gamma(m) \Gamma(m+0.5)}\left(4+\frac{2 E N_{2}^{2} \Omega}{m \sigma_{n}^{2}}\right)^{-m}, \\
& \nu_{2} \triangleq 2 \sigma_{n}^{2}\left(1+2 m \frac{\sigma_{n}^{2}}{E N_{2}^{2} \Omega}\right),
\end{aligned}
$$

and $\Phi$ denoting a confluent hypergeometric function given by $[44]$ :

$$
\begin{aligned}
\Phi\left(\beta_{1}, \beta_{2} ; x\right)= & 1+\frac{\beta_{1}}{\beta_{2}} \frac{x}{1 !}+\frac{\beta_{1}\left(\beta_{1}+1\right)}{\beta_{2}\left(\beta_{2}+1\right)} \frac{x^{2}}{2 !} \\
& +\frac{\beta_{1}\left(\beta_{1}+1\right)\left(\beta_{1}+2\right)}{\beta_{2}\left(\beta_{2}+1\right)\left(\beta_{2}+2\right)} \frac{x^{3}}{3 !}+\cdots .
\end{aligned}
$$

Note that the p.d.f. of $N_{2} \sqrt{E} d|\alpha|+\eta, p_{2}(z)$ is obtained from (14), (24), and the fact that $d$ is \pm 1 with equal probability.

After some manipulation, the TOA estimation rule can be expressed as

$$
\begin{aligned}
\hat{k}=\arg \max _{k \in \mathcal{U}}\left\{\log \left[\nu_{1} \Phi\left(m, 0.5 ; \frac{z_{k}^{2}}{v_{2}}\right)\right]\right. \\
\left.+\sum_{i=k+1}^{n_{\mathrm{f}}} \log \left[p \nu_{1} \Phi\left(m, 0.5 ; \frac{z_{i}^{2}}{v_{2}}\right)+1-p\right]\right\} .
\end{aligned}
$$

Note that this estimation rule does not require any threshold setting, since it obtains the TOA estimate as the chip index that maximizes the decision variable in (28). 


\subsection{Additional tests}

The formulation in (12) assumes that the block always starts with noise-only components, and then the signal paths start to arrive. However, in practice, there can be cases in which the first step chooses a block consisting of all noise components. By combining a large number of frames, that is, by choosing a large $N_{1}$ in (7), the probability of this event can be reduced considerably. However, very large $N_{1}$ also increases the estimation time. Hence, there is a tradeoff between the estimation error and the estimation time. In order to prevent erroneous TOA estimation when a noiseonly block is chosen, a one-sided test can be applied using the known distribution of the noise outputs. Since the noise outputs have a Gaussian distribution, the test reduces to the comparison of the average energy of the outputs after the estimated change instant against a threshold. In other words, if $\left(1 /\left(n_{\mathrm{f}}-\hat{k}+1\right)\right) \sum_{i=\hat{k}}^{n_{\mathrm{f}}} z_{i}^{2}<\delta_{1}$, the block is considered as a noise-only block and the two-step algorithm is run again.

Another improvement of the algorithm can be obtained by checking if the block consists of all signal paths, that is, the TOA is prior to the current block. Again, by following a one-sided test approach, we can check the average energy of the correlation outputs before the estimated TOA against a threshold and detect an all-signal block if the threshold is exceeded. However, for very small values of the TOA estimate $\hat{k}$, there can be a significant probability that the first signal path arrives before the current observation region since the distribution of the correlation output after the first path includes both the noise distribution and the signalplus-noise distribution with some probabilities as shown in (13). Hence, the test may fail although the block is an allsignal block. Therefore, some additional correlation outputs before $\hat{k}$ can be employed as well, when calculating the average power before the TOA estimate. In other words, if $\left(1 /\left(\hat{k}-n_{\mathrm{s}}+M_{3}\right)\right) \sum_{i=n_{\mathrm{s}}-M_{3}}^{\hat{k}-1} z_{i}^{2}>\delta_{2}$, the block is considered as an all-signal block, where $M_{3} \geq 0$ additional outputs are used depending on $\hat{k}$. When it is determined that the block consists of all signal outputs, the TOA is expected to be in one of the previous blocks. Therefore, the uncertainty region is shifted backwards, and the change detection algorithm is repeated.

\subsection{Probability of block detection}

In the proposed two-step TOA estimator, determination of the block that contains the first signal path carries significant importance. Therefore, in this section, the probability of selecting the correct block is analyzed in detail.

Let the received signal in the $i$ th block of the $j$ th frame be denoted by $r_{i, j}(t)$, that is,

$$
r_{i, j}(t) \doteq \begin{cases}r(t), & t \in\left[j T_{\mathrm{f}}+i T_{\mathrm{b}}, j T_{\mathrm{f}}+(i+1) T_{\mathrm{b}}\right], \\ 0, & \text { otherwise }\end{cases}
$$

for $i=0,1, \ldots, N_{\mathrm{b}}-1$, and $j=0,1, \ldots, N_{1}-1$. Under the assumption that the channel impulse response does not change during at least $N_{1}$ frame intervals, $r_{i, j}(t)$ can be expressed as

$$
r_{i, j}(t)=s_{i}(t)+n_{i, j}(t),
$$

where $s_{i}(t)$ is the signal part in the $i$ th block, and $n_{i, j}(t)$ is the noise in the $i$ th block of the $j$ th frame. Note that due to the static channel assumption, the signal part is identical for the $i$ th block of all $N_{1}$ frames. In addition, the noise components are independent for different block and/or frame indices.

From (29) and (30), the signal energy in (8) can be expressed as

$$
Y_{i, j}=\int_{-\infty}^{\infty}\left|r_{i, j}(t)\right|^{2} d t
$$

which becomes

$$
Y_{i, j}=\int_{-\infty}^{\infty}\left|n_{i, j}(t)\right|^{2} d t
$$

for noise-only blocks, and

$$
Y_{i, j}=\int_{-\infty}^{\infty}\left|s_{i}(t)+n_{i, j}(t)\right|^{2} d t,
$$

for signal-plus-noise blocks, that is, for blocks that contain some signal components in addition to noise. It can be shown that $Y_{i, j}$ has a central or noncentral chi-square distribution depending on the type of the block. Let $\mathscr{B}_{n}$ and $\mathscr{B}_{s}$ represent the sets of block indices for noise-only and signal-plus-noise blocks, respectively. Then,

$$
Y_{i, j} \sim \begin{cases}\chi_{n}^{2}(0), & i \in \mathscr{B}_{n}, \\ \chi_{n}^{2}\left(\varepsilon_{i}\right), & i \in \mathscr{B}_{s},\end{cases}
$$

where $n$ is the approximate dimensionality of the signal space, which is obtained from the time-bandwidth product [47]; $\varepsilon_{i}$ is the energy of the signal in the ith block; $\varepsilon_{i}=\int\left|s_{i}(t)\right|^{2} d t$; and $\chi_{n}^{2}(\varepsilon)$ denotes a noncentral chi-square distribution with $n$ degrees of freedom and a noncentrality parameter of $\varepsilon$. Clearly, $\chi_{n}^{2}(\varepsilon)$ reduces to a central chi-square distribution with $n$ degrees of freedom for noise-only blocks for which $\varepsilon=0$.

As expressed in (7), each decision variable for block estimation is obtained by adding signal energy from $N_{1}$ frames. From the fact that the sum of i.i.d. noncentral chisquare random variables with $n$ degrees of freedom and with noncentrality parameter $\varepsilon$ results in another noncentral chisquare random variable with $N_{1} n$ degrees of freedom and noncentrality parameter $N_{1} \varepsilon$, the probability distribution of $Y_{i}$ in (7) can be expressed as

$$
Y_{i}=\sum_{j=0}^{N_{1}-1} Y_{i, j} \sim \begin{cases}\chi_{N_{1} n}^{2}(0), & i \in \mathcal{B}_{n}, \\ \chi_{N_{1} n}^{2}\left(N_{1} \varepsilon_{i}\right), & i \in \mathcal{B}_{s} .\end{cases}
$$

The probability that the TOA estimator selects the $l$ th block, which is a signal-plus-noise block, as the block that contains the first signal path is given by

$$
P_{\mathrm{D}}^{l}=\operatorname{Pr}\left\{Y_{l}>Y_{i}, \forall i \neq l\right\}
$$


for $l \in \mathscr{B}_{s}$, which can be expressed as

$$
P_{\mathrm{D}}^{l}=\int_{0}^{\infty} p_{Y_{l}}(y) \prod_{i \in \mathscr{B}_{s} \backslash\{l\}} \operatorname{Pr}\left\{Y_{i}<y\right\} \prod_{j \in \mathscr{B}_{n}} \operatorname{Pr}\left\{Y_{j}<y\right\} d y,
$$

where $p_{Y_{l}}(y)$ represents the p.d.f. of the signal energy in the lth block. Since the energies of the noise-only blocks are i.i.d., (37) becomes

$$
P_{\mathrm{D}}^{l}=\int_{0}^{\infty} p_{Y_{l}}(y)\left(\operatorname{Pr}\left\{Y_{j}<y\right\}\right)^{\left|\mathcal{B}_{n}\right|} \prod_{i \in \mathscr{B}_{s} \backslash\{l\}} \operatorname{Pr}\left\{Y_{i}<y\right\} d y,
$$

where $\left|\mathscr{B}_{n}\right|$ denotes the number of elements in set $\mathscr{B}_{n}$, and $j$ can be any value from $\mathscr{B}_{n}$. (It is also observed from (35) that the p.d.f. of energy in a noise-only block does not depend on the index of the block.)

From (35), (38) can be obtained, after some manipulation, as in the appendix:

$$
\begin{aligned}
P_{\mathrm{D}}^{l}=\frac{e^{-N_{1} \varepsilon /\left(2 \sigma^{2}\right)}}{\left(2 \sigma^{2}\right)^{\left|\mathcal{B}_{s}\right|}} \int_{0}^{\infty} & f_{l}(y)\left(1-e^{-y /\left(2 \sigma^{2}\right)} \sum_{k=0}^{N_{1} n / 2-1} \frac{1}{k !}\left(\frac{y}{2 \sigma^{2}}\right)^{k}\right)^{\left|\mathcal{B}_{n}\right|} \\
& \times \prod_{i \in \mathcal{B}_{s} \backslash\{l\}} \int_{0}^{y} f_{i}(x) d x d y,
\end{aligned}
$$

where $N_{1} n$ is assumed to be an even number; $\varepsilon=\sum_{i \in \mathcal{B}_{s}} \varepsilon_{i}$ represents the total signal energy; and

$$
f_{l}(y)=e^{-y /\left(2 \sigma^{2}\right)}\left(\frac{y}{N_{1} \varepsilon}\right)^{\left(N_{1} n-2\right) / 4} \mathrm{I}_{N_{1} n / 2-1}\left(\frac{\sqrt{N_{1} \varepsilon_{l} y}}{\sigma^{2}}\right)
$$

with

$$
\mathrm{I}_{\kappa}(x)=\sum_{i=0}^{\infty} \frac{(x / 2)^{\kappa+2 i}}{i ! \Gamma(\kappa+i+1)}, \quad x \geq 0
$$

representing the $\kappa$ th-order modified Bessel function of the first kind, and $\Gamma(\cdot)$ denoting the gamma function [48].

In the presence of a single signal-plus-noise block, that is, $\mathscr{B}_{s}=\{l\},(39)$ reduces to

$$
P_{\mathrm{D}}^{l}=\frac{e^{-N_{1} \varepsilon_{l} /\left(2 \sigma^{2}\right)}}{2 \sigma^{2}} \int_{0}^{\infty} f_{l}(y)\left(1-e^{-y /\left(2 \sigma^{2}\right)} \sum_{k=0}^{N_{1} n / 2-1} \frac{1}{k !}\left(\frac{y}{2 \sigma^{2}}\right)^{k}\right)^{\left|\mathcal{B}_{n}\right|} d y,
$$

which can be evaluated easily via numerical integration. However, in the presence of multiple signal-plus-noise blocks, numerical integration to calculate $P_{\mathrm{D}}^{l}$ from (39) and (40) can have high computational complexity. Therefore, a Monte-Carlo approach can be followed, by generating a number of noncentral chi-square distributed samples, and by approximating the expectation operation in (38) by the sample mean of the inner probability terms. Although the probability of detecting block $l$ can be calculated exactly based on (39) and (40), a simpler expression can be obtained by means of Gaussian approximation for a large number of frames. In other words, for large values of $N_{1}, Y_{i}$ in (7) can be approximated by a Gaussian random variable.

From (34), the Gaussian approximation can be obtained as

$$
\begin{aligned}
& Y_{i} \\
& =\sum_{j=0}^{N_{1}-1} Y_{i, j} \sim \begin{cases}\mathcal{N}\left(N_{1} n \sigma^{2}, 2 N_{1} n \sigma^{4}\right), & i \in \mathcal{B}_{n}, \\
\mathcal{N}\left(N_{1}\left(n \sigma^{2}+\varepsilon_{i}\right), 2 N_{1} \sigma^{2}\left(n \sigma^{2}+2 \varepsilon_{i}\right)\right), & i \in \mathcal{B}_{s} .\end{cases}
\end{aligned}
$$

Then, the probabilities that the energy of the lth block is larger than that of the other signal-plus-noise blocks or than the noise-only blocks are given, respectively, by

$$
\operatorname{Pr}\left\{Y_{i}<y\right\} \approx Q\left(\frac{N_{1}\left(n \sigma^{2}+\varepsilon_{i}\right)-y}{\sqrt{2 N_{1} \sigma^{2}\left(n \sigma^{2}+2 \varepsilon_{i}\right)}}\right)
$$

for $i \in \mathscr{B}_{s} \backslash\{l\}$, and

$$
\operatorname{Pr}\left\{Y_{j}<y\right\} \approx Q\left(\frac{N_{1} n \sigma^{2}-y}{\sigma^{2} \sqrt{2 N_{1} n}}\right)
$$

for $j \in B_{n}$, where $Q(x)=(1 / \sqrt{2 \pi}) \int_{x}^{\infty} e^{-t^{2} / 2} d t$ represents the $Q$-function. Note that the detection probability in (38) can be calculated easily from (44) and (45) via numerical integration techniques. In addition, as will be investigated in Section 4, the Gaussian approximation is quite accurate for practical signal parameters.

Since the index of the block that includes the first signal path is denoted by $k_{b}$ in Section 3, the probability that the correct block is selected is given by $P_{\mathrm{D}}^{k_{b}}$, which can be obtained from (38)-(45). If the TOA estimator searches both the selected block and the previous block in order to increase the probability that the first signal path is included in the search space of the second step, then the probability of including the first signal path in the search space of the second step is given by $P_{\mathrm{D}}^{k_{b}}+P_{\mathrm{D}}^{k_{b}+1}$.

\section{SIMULATION RESULTS}

In this section, numerical studies and simulations are performed in order to evaluate the expressions in Section 3.4, and to investigate the performance of the proposed TOA estimator over realistic IEEE 802.15.4a channel models [43, 49].

First, the expressions in Section 3.4 for probability of block detection are investigated. Consider a scenario with $N_{\mathrm{b}}=10$ blocks, all of which are noise-only blocks except for the fifth one. Also, the degrees of freedom for each energy sample, $n$ in (34), are taken to be 10. In Figure 3, the probabilities of block detection are plotted versus SNR for $N_{1}=5$ and $N_{1}=25$, where $N_{1}$ is the number of frames over which the energy samples are combined. SNR is defined as the ratio between the total signal energy $\varepsilon$ in the blocks and $\sigma^{2}$ (Section 3.4). It is observed that the exact expression and the one based on Gaussian approximation yield very close values. Especially, for $N_{1}=25$, the results are in very good 


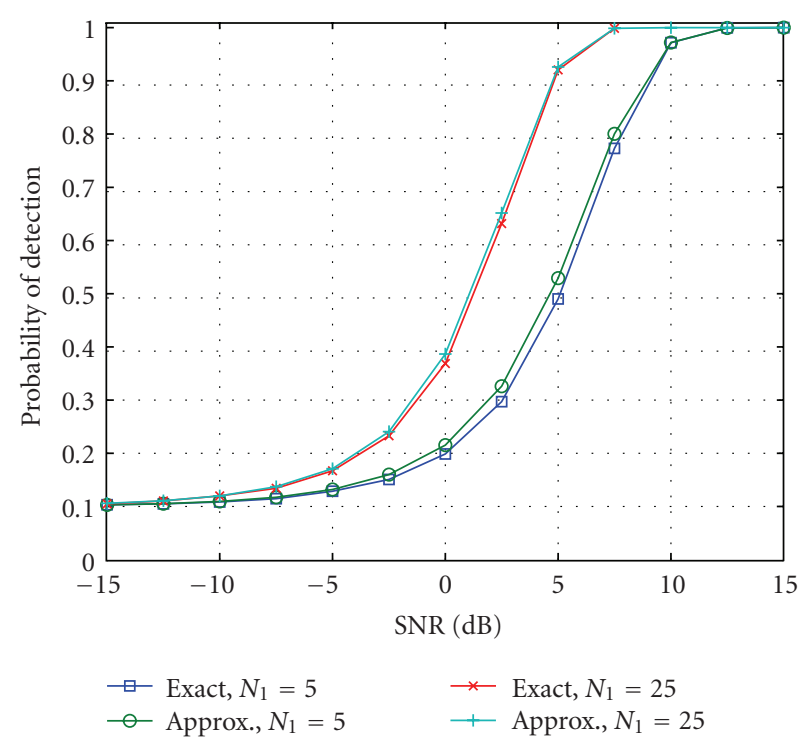

FIgURE 3: Probability of block detection versus SNR for $N_{\mathrm{b}}=10$, $n=10$, and $\varepsilon_{i}=0 \quad \forall i \neq 5$.

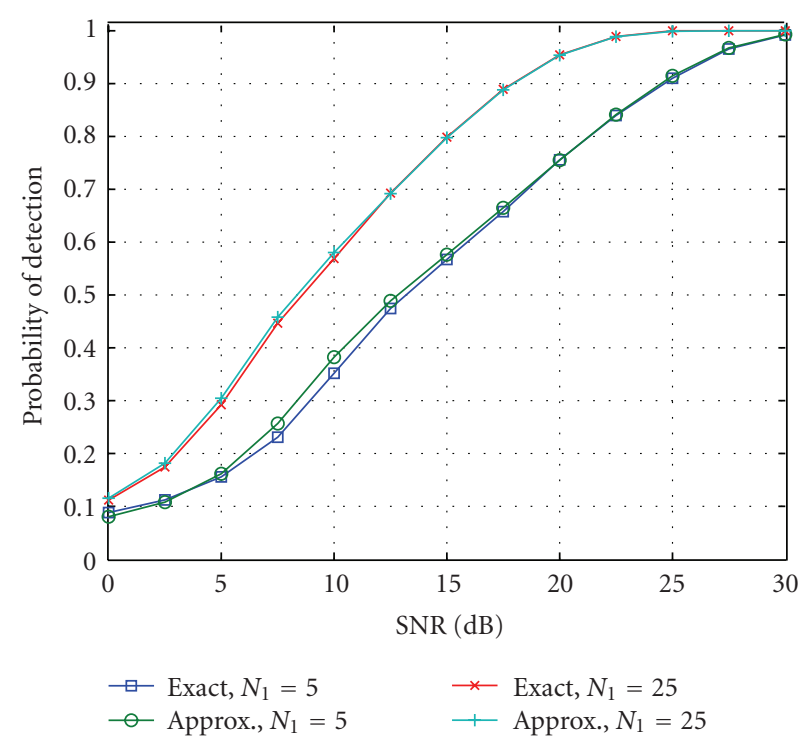

FIgURE 4: Probability of block detection versus SNR for $N_{\mathrm{b}}=20$, $n=5$, and $\boldsymbol{\varepsilon}=\left[\begin{array}{llllll}3 & 2.5 & 2 & 1.25 & 0.5 & \mathbf{0}_{15}\end{array}\right]$.

agreement, as the Gaussian approximation becomes more accurate as $N_{1}$ increases.

In Figure 4, the probability of block detections are plotted versus SNR for $N_{\mathrm{b}}=20, n=5$, and $\boldsymbol{\varepsilon}=$ $\left[\begin{array}{llllll}3 & 2.5 & 2 & 1.25 & 0.5 & \mathbf{0}_{15}\end{array}\right]$, where $\boldsymbol{\varepsilon}=\left[\begin{array}{lll}\varepsilon_{1} \cdots \varepsilon_{\mathrm{N}_{\mathrm{b}}}\end{array}\right]$, and $\mathbf{0}_{15}$ represents a row vector of 15 zeros. From the plot, it is observed that the exact and approximate curves are in good agreement as in the previous case. Also, due to the presence of multiple signal blocks with close energy levels, higher SNR values, than those in the previous case, are needed for reliable detection of the first block in this scenario.

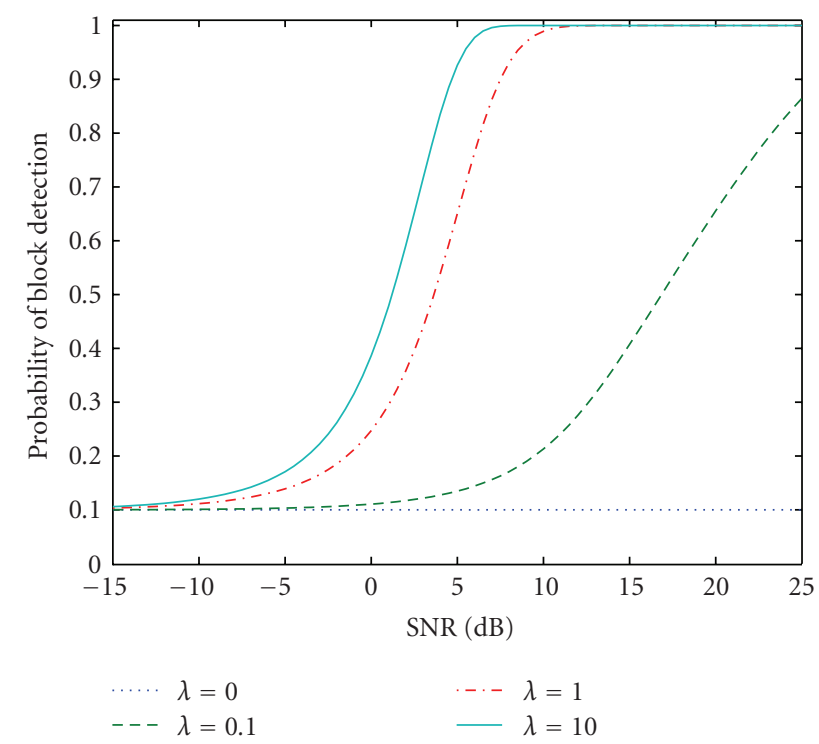

FIGURE 5: Probability of block detection versus SNR for $\varepsilon_{i}=e^{-\lambda(i-1)}$ for $i=1, \ldots, N_{\mathrm{b}}, n=10, N_{1}=25$, and $N_{\mathrm{b}}=10$.

Next, the block energies are modeled as exponentially decaying, $\varepsilon_{i}=e^{-\lambda(i-1)}$ for $i=1, \ldots, N_{\mathrm{b}}$, and the block detection probabilities are obtained for various decay factors, for $n=10, N_{1}=25$, and $N_{\mathrm{b}}=10$. In Figure 5, better detection performance is observed as the decay factor increases. In other words, if the energy of the first block is considerably larger than the energies of the other blocks, the probability of block detection increases. At the extreme case in which all the blocks have the same energy, the probability converges to 0.1 , which is basically equal to the probability of selecting one of the 10 blocks in a random fashion.

In order to investigate the performance of the proposed estimator, residential and office environments with both lineof-sight (LOS) and nonline-of-sight (NLOS) situations are considered according to the IEEE 802.15.4a channel models [43]. In the simulation scenario, the signal bandwidth is $7.5 \mathrm{GHz}$ and the frame time of the transmitted training sequence is 300 nanoseconds. Hence, an uncertainty region consisting of 2250 chips is considered, and that region is divided into $N_{\mathrm{b}}=50$ blocks. In the proposed algorithm, the numbers of pulses, over which the correlations are taken in the first and second steps, are given by $N_{1}=50$ and $N_{2}=25$, respectively. Also $M_{1}=180$ additional chips prior to the uncertainty region determined by the first step are included in the second step. The estimator is assumed to have 10 parallel correlators for the second step. In a practical setting, the estimator can use the correlators of a Rake receiver that is already present for the signal demodulation, and 10 is a conservative value in this sense.

From the simulations, it is obtained that each TOA estimation takes about 1 millisecond ( 0.92 millisecond to be more precise). (Since we do not employ any additional tests after the TOA estimate, which are described in Section 3.3, and use the same parameters for all the channel models, the estimation time is the same for all the channel realizations.) 


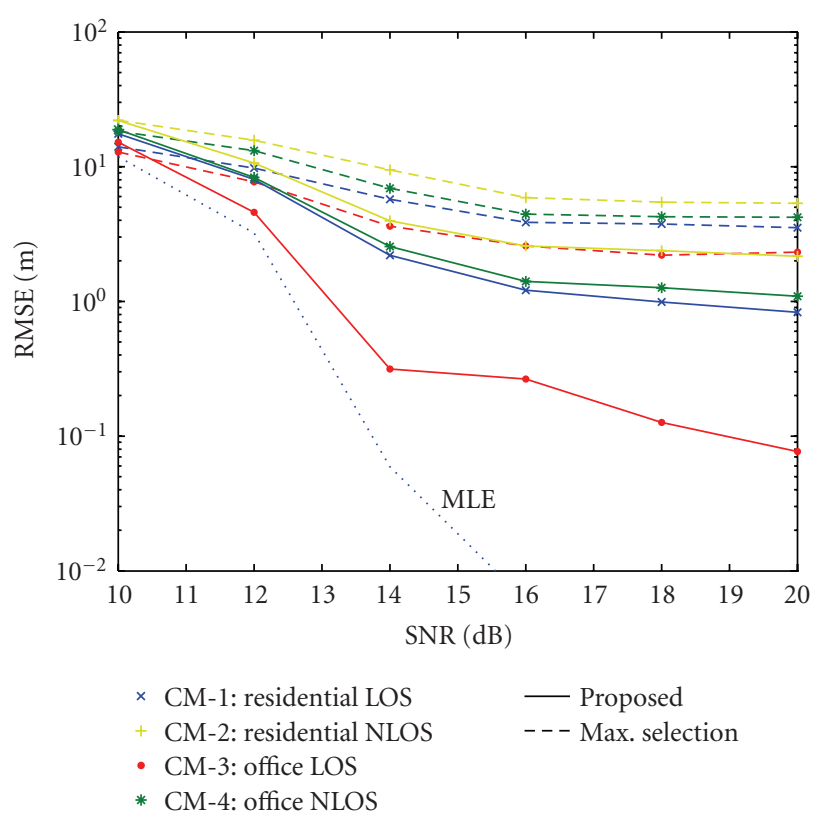

FIGURE 6: RMSE versus SNR for the proposed and the conventional maximum (peak) selection algorithms.

In order to have a fair comparison with the conventional correlation-based peak selection algorithm, a training signal duration of 1 millisecond is considered for that algorithm as well. For both algorithms, frame-rate sampling is assumed. In Figure 6, the root-mean-square errors are plotted versus SNR for the proposed and the conventional algorithms under four different channel conditions. Due to the different characteristics of the channels in residential and office environments, the estimates are better in the office environment. Namely, the delay spread is smaller in the channel models for the office environment. Moreover, as expected, the NLOS situations cause increase in the RMSE values. Comparison of the two algorithms reveal that the proposed algorithm can provide better accuracy than the conventional one. Especially, at high SNR values the proposed algorithm can provide less than a meter accuracy for LOS channels and about 2 meters accuracy for NLOS channels. In addition to the conventional and the proposed approaches, the maximum likelihood estimator (MLE) is also illustrated in Figure 6 as a theoretical limit for CM3. For the MLE, it is assumed that Nyquist-rate samples of the signal can be obtained over two frames and the channel coefficients are known. Note that due to the impractical assumptions related to the MLE, the lower limit provided by the MLE is not tight. Therefore, it is concluded that more realistic theoretical limits (e.g., CRLB) based on lowrate noncoherent and coherent signal samples need to be obtained, which are a topic of future research.

Note that one disadvantage of the conventional approach is that it needs to search for TOA in every chip position one by one. However, the proposed algorithm first employs coarse TOA estimation, and therefore it can perform fine TOA estimation only in a smaller uncertainty region. In

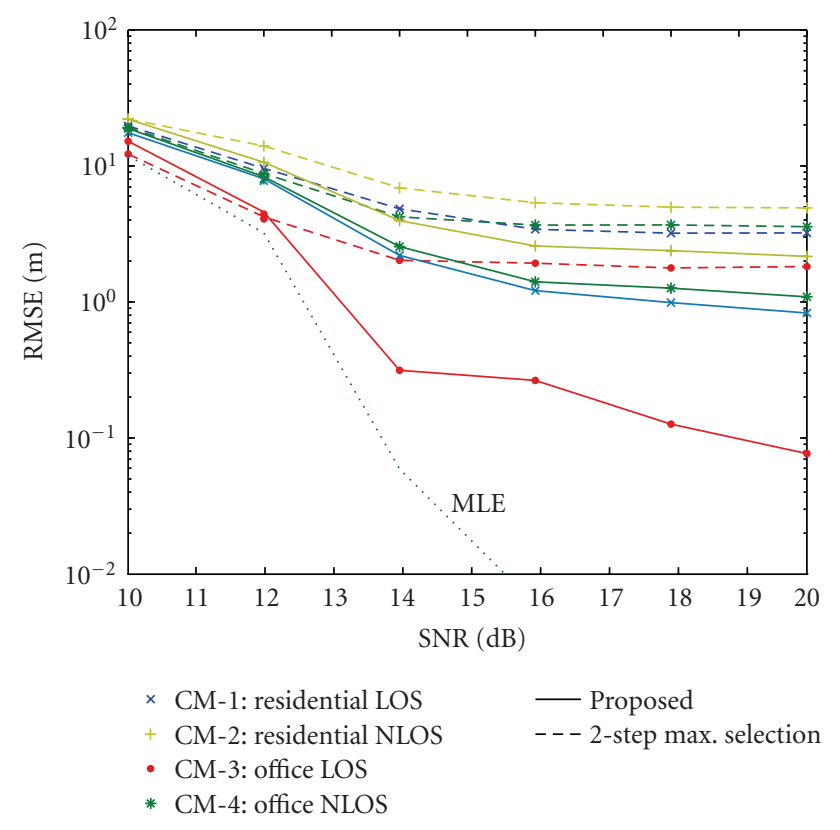

FIGURE 7: RMSE versus SNR for the proposed and the two-step peak selection algorithms.

order to investigate how much the conventional algorithm can be improved by applying a similar two-step approach, a modified version of the conventional algorithm is considered, which first employs the coarse TOA estimation (via energy detection), and then performs the conventional peak selection in the second step. Figure 7 compares the proposed algorithm with the modified version of the conventional algorithm. Note from Figures 6 and 7 that the performance of the conventional algorithm is slightly enhanced by employing a two-step approach, since correlation outputs can be obtained more reliably over the 1 millisecond training signal interval for the latter. In other words, more time can be allocated to the chip positions around the TOA by applying the coarse TOA estimation first. However, the performance is still considerably worse than that of the proposed approach, since the peak selection in the conventional approach performs significantly worse than the proposed change detection technique.

Finally, note that for the proposed algorithm, the same parameters are used for all the channel models. More accurate results can be obtained by employing different parameters in different scenarios. In addition, by applying additional tests described in Section 3.3, the accuracy can be enhanced even further.

\section{CONCLUSIONS}

In this paper, we have proposed a two-step TOA estimation algorithm, where the first step uses RSS measurements to quickly obtain a coarse TOA estimate, and the second step uses a change detection approach to estimate the fine TOA of the signal. The proposed scheme relies on low-rate correlation outputs, but still obtains a considerably accurate 
TOA estimate in a reasonable time interval, which makes it quite practical for realistic UWB systems. Simulations have been performed to analyze the performance of the proposed TOA estimator, and the comparisons with the conventional TOA estimation techniques have been presented.

\section{APPENDIX}

\section{A. DERIVATION OF (39)}

Since the energy is distributed according to noncentral chisquare distribution for signal-plus-noise blocks, as specified by (35), $p_{Y_{l}}(y)$ in (38) is given by

$$
p_{Y_{l}}(y)=\frac{1}{2 \sigma^{2}}\left(\frac{y}{N_{1} \varepsilon_{l}}\right)^{\left(N_{1} n-2\right) / 4} e^{-\left(y+N_{1} \varepsilon_{l}\right) / 2 \sigma^{2}} \mathrm{I}_{N_{1} n / 2-1}\left(\frac{\sqrt{N_{1} \varepsilon_{l} y}}{\sigma^{2}}\right)
$$

for $y \geq 0$, where $\mathrm{I}_{\kappa}(\cdot)$ is as defined in (41). Similarly, $\operatorname{Pr}\left\{Y_{i}<\right.$ $y$ \} can be obtained from the following expression:

$$
\begin{aligned}
& \operatorname{Pr}\left\{Y_{i}<y\right\} \\
& =\frac{1}{2 \sigma^{2}} \int_{0}^{y}\left(\frac{x}{N_{1} \varepsilon_{i}}\right)^{\left(N_{1} n-2\right) / 4} e^{-\left(x+N_{1} \varepsilon_{i}\right) / 2 \sigma^{2}} \mathrm{I}_{N_{1} n / 2-1}\left(\frac{\sqrt{N_{1} \varepsilon_{i} x}}{\sigma^{2}}\right) d x
\end{aligned}
$$

for $i \in \mathcal{B}_{s}$.

Since the energy is distributed according to a central chi-square distribution for noise-only blocks, as specified by (35), the $\operatorname{Pr}\left\{Y_{j}<y\right\}$ is given by

$$
\operatorname{Pr}\left\{Y_{j}<y\right\}=\frac{1}{2^{N_{1} n / 2} \sigma^{N_{1} n} \Gamma\left(N_{1} n / 2\right)} \int_{0}^{y} x^{N_{1} n / 2-1} e^{-x / 2 \sigma^{2}} d x
$$

for $j \in \mathcal{B}_{n}$, where $\Gamma(\cdot)$ represents the gamma function.

For even values of $N_{1} n,(\mathrm{~A} .3)$ can be expressed as [48]:

$$
\operatorname{Pr}\left\{Y_{j}<y\right\}=1-e^{-y / 2 \sigma^{2}} \sum_{k=0}^{N_{1} n / 2-1} \frac{1}{k !}\left(\frac{y}{2 \sigma^{2}}\right)^{k} .
$$

Then, from (A.1), (A.2), and (A.4), (38) can be expressed as in (39) and (40), after some manipulation.

\section{ACKNOWLEDGMENTS}

This work was supported in part by the European Commission in the framework of the FP7 Network of Excellence in Wireless COMmunications NEWCOM++ (Contract no. 216715), and in part by the U. S. National Science Foundation under Grants ANI-03-38807 and CNS-06-25637. Part of this work was presented at the 13th European Signal Processing Conference, Antalya, Turkey, September, 2005.

\section{REFERENCES}

[1] U. S. Federal Communications Commission, FCC 02-48: First Report and Order.
[2] IEEE P802.15.4a/D4 (Amendment of IEEE Std 802.15.4), "Part 15.4: Wireless Medium Access Control (MAC) and Physical Layer (PHY) Specifications for Low-Rate Wireless Personal Area Networks (LRWPANs)," July 2006.

[3] S. Gezici, Z. Tian, G. B. Giannakis, et al., "Localization via ultra-wideband radios: a look at positioning aspects of future sensor networks," IEEE Signal Processing Magazine, vol. 22, no. 4, pp. 70-84, 2005.

[4] A. F. Molisch, Y. G. Li, Y.-P. Nakache, et al., "A lowcost time-hopping impulse radio system for high data rate transmission," EURASIP Journal on Applied Signal Processing, vol. 2005, no. 3, pp. 397-412, 2005.

[5] L. Yang and G. B. Giannakis, "Ultra-wideband communications: an idea whose time has come," IEEE Signal Processing Magazine, vol. 21, no. 6, pp. 26-54, 2004.

[6] ECMA-368, "High Rate Ultra Wideband PHY and MAC Standard," 2nd edition, December 2007, http://www.ecmainternational.org/publications/files/ECMA-ST/ECMA-368 .pdf.

[7] M. Z. Win and R. A. Scholtz, "Impulse radio: how it works," IEEE Communications Letters, vol. 2, no. 2, pp. 36-38, 1998.

[8] M. Z. Win and R. A. Scholtz, "Ultra-wide bandwidth timehopping spread-spectrum impulse radio for wireless multipleaccess communications," IEEE Transactions on Communications, vol. 48, no. 4, pp. 679-691, 2000.

[9] F. Ramirez-Mireles, "On the performance of ultra-wideband signals in Gaussian noise and dense multipath," IEEE Transactions on Vehicular Technology, vol. 50, no. 1, pp. 244249, 2001.

[10] R. A. Scholtz, "Multiple access with time-hopping impulse modulation," in Proceedings of the IEEE Military Communications Conference (MILCOM '93), vol. 2, pp. 447-450, Boston, Mass, USA, October 1993.

[11] D. Cassioli, M. Z. Win, and A. F. Molisch, "The ultra-wide bandwidth indoor channel: from statistical model to simulations," IEEE Journal on Selected Areas in Communications, vol. 20, no. 6, pp. 1247-1257, 2002.

[12] K. Siwiak and J. Gabig, "IEEE 802.15.4IGa Informal Call for Application Response, Contribution\#11," Doc.: IEEE 802.15-04/266r0, July 2003, http://www.ieee802.org/15/pub/ TG4a.html.

[13] S. Gezici, Z. Sahinoglu, H. Kobayashi, and H. V. Poor, "Ultra wideband geolocation," in Ultra Wideband Wireless Communications, H. Arslan, Z. N. Chen, and M.-G. Di Benedetto, Eds., John Wiley \& Sons, New York, NY, USA, 2006.

[14] V. Lottici, A. D'Andrea, and U. Mengali, "Channel estimation for ultra-wideband communications," IEEE Journal on Selected Areas in Communications, vol. 20, no. 9, pp. 1638-1645, 2002.

[15] M. Z. Win and R. A. Scholtz, "Characterization of ultrawide bandwidth wireless indoor channels: a communicationtheoretic view," IEEE Journal on Selected Areas in Communications, vol. 20, no. 9, pp. 1613-1627, 2002.

[16] J.-Y. Lee and R. A. Scholtz, "Ranging in a dense multipath environment using an UWB radio link," IEEE Journal on Selected Areas in Communications, vol. 20, no. 9, pp. 1677$1683,2002$.

[17] V. S. Somayazulu, J. R. Foerster, and S. Roy, "Design challenges for very high data rate UWB systems," in Proceedings of the Conference Record of the 36th Asilomar Conference on Signals, Systems and Computers, vol. 1, pp. 717-721, Pacific Grove, Calif, USA, November 2002.

[18] J. Caffery Jr., Wireless Location in CDMA Cellular Radio Systems, Kluwer Academic Publishers, Boston, Mass, USA, 2000 . 
[19] E. A. Homier and R. A. Scholtz, "Rapid acquisition of ultra-wideband signals in the dense multipath channel," in Proceedings of the IEEE Conference on Ultra Wideband Systems and Technologies (UWBST '02), pp. 105-109, Baltimore, Md, USA, May 2002.

[20] D. Dardari, C.-C. Chong, and M. Z. Win, "Analysis of threshold-based TOA estimators in UWB channels," in Proceedings of the 14th European Signal Processing Conference (EUSIPCO '06), Florence, Italy, September 2006.

[21] I. Guvenc and Z. Sahinoglu, "Threshold-based TOA estimation for impulse radio UWB systems," in Proceedings of the IEEE International Conference on Ultra-Wideband (ICU '05), pp. 420-425, Zurich, Switzerland, September 2005.

[22] K. Yu and I. Oppermann, "Performance of UWB position estimation based on time-of-arrival measurements," in Proceedings of the IEEE Conference on Ultra Wideband Systems and Technologies (UWBST '04), pp. 400-404, Kyoto, Japan, May 2004.

[23] L. Yang and G. B. Giannakis, "Timing ultra-wideband signals with dirty templates," IEEE Transactions on Communications, vol. 53, no. 11, pp. 1952-1963, 2005.

[24] C. Falsi, D. Dardari, L. Mucchi, and M. Z. Win, "Time of arrival estimation for UWB localizers in realistic environments," EURASIP Journal on Applied Signal Processing, vol. 2006, Article ID 32082, 13 pages, 2006.

[25] R. A. Scholtz and J.-Y. Lee, "Problems in modeling UWB channels," in Proceedings of the Conference Record of the 36th Asilomar Conference on Signals, Systems and Computers, vol. 1, pp. 706-711, Pacific Grove, Calif, USA, November 2002.

[26] L. Yang and G. B. Giannakis, "Low-complexity training for rapid timing acquisition in ultra wideband communications," in Proceedings of the IEEE Global Telecommunications Conference (GLOBECOM '03), vol. 2, pp. 769-773, San Francisco, Calif, USA, December 2003.

[27] L. Yang and G. B. Giannakis, "Blind UWB timing with a dirty template," in Proceedings of the IEEE International Conference on Acoustics, Speech and Signal Processing (ICASSP'04), vol. 4, pp. 509-512, Montreal, Canada, May 2004.

[28] L. Yang and G. B. Giannakis, "Ultra-wideband communications: an idea whose time has come," IEEE Signal Processing Magazine, vol. 21, no. 6, pp. 26-54, 2004.

[29] Y.-P. Nakache and A. F. Molisch, "Spectral shape of UWB signals-influence of modulation format, multiple access scheme and pulse shape," in Proceedings of the 57th IEEE Semiannual Vehicular Technology Conference (VTC '03), vol. 4, pp. 2510-2514, Jeju, Korea, April 2003.

[30] S. Gezici, Z. Sahinoglu, H. Kobayashi, and H. V. Poor, "Ultrawideband impulse radio systems with multiple pulse types," IEEE Journal on Selected Areas in Communications, vol. 24, no. 4, part 1, pp. 892-898, 2006.

[31] E. Fishler and H. V. Poor, "On the tradeoff between two types of processing gains," IEEE Transactions on Communications, vol. 53, no. 10, pp. 1744-1753, 2005.

[32] S. Gezici, A. F. Molisch, H. V. Poor, and H. Kobayashi, "The trade-off between processing gains of an impulse radio UWB system in the presence of timing jitter," IEEE Transactions on Communications, vol. 55, no. 8, pp. 1504-1515, 2007.

[33] Z. Sahinoglu and S. Gezici, "Ranging in the IEEE 802.15.4a standard," in Proceedings of the IEEE Annual Wireless and Microwave Technology Conference (WAMICON '06), pp. 1-5, Clearwater Beach, Fla, USA, December 2006.

[34] D. Lee and L. B. Milstein, "Comparison of multicarrier DSCDMA broadcast systems in a multipath fading channel,"
IEEE Transactions on Communications, vol. 47, no. 12, pp. 1897-1904, 1999.

[35] W. Xu and L. B. Milstein, "On the performance of multicarrier RAKE systems," IEEE Transactions on Communications, vol. 49, no. 10, pp. 1812-1823, 2001.

[36] S. Gezici, H. Kobayashi, H. V. Poor, and A. F. Molisch, "Performance evaluation of impulse radio UWB systems with pulse-based polarity randomization," IEEE Transactions on Signal Processing, vol. 53, no. 7, pp. 2537-2549, 2005.

[37] S. R. Aedudodla, S. Vijayakumaran, and T. F. Wong, “Timing acquisition in ultra-wideband communication systems," IEEE Transactions on Vehicular Technology, vol. 54, no. 5, pp. 15701583, 2005.

[38] Y. Shimizu and Y. Sanada, "Accuracy of relative distance measurement with ultra wideband system," in Proceedings of the IEEE Conference on Ultra Wideband Systems and Technologies (UWBST '03), pp. 374-378, Reston, Va, USA, November 2003.

[39] Z. Yuanjin, C. Rui, and L. Yong, "A new synchronization algorithm for UWB impulse radio communication systems," in Proceedings of the 9th IEEE Singapore International Conference on Communication Systems (ICCS '04), pp. 25-29, Singapore, September 2004.

[40] X. Cheng and A. Dinh, "Ultrawideband synchronization in dense multi-path environment," in Proceedings of the IEEE Pacific RIM Conference on Communications, Computers, and Signal Processing (PACRIM '05), pp. 29-32, Victoria, Canada, August 2005.

[41] L. Ma, A. Duel-Hallen, and H. Hallen, "Physical modeling and template design for UWB channels with per-path distortion," in Proceedings of the IEEE Military Communications Conference (MILCOM '07), pp. 1-7, Washington, DC, USA, October 2007.

[42] R. D. Wilson and R. A. Scholtz, "Template estimation in ultrawideband radio," in Proceedings of the Conference Record of the 36th Asilomar Conference on Signals, Systems and Computers, vol. 2, pp. 1244-1248, Pacific Grove, Calif, USA, November 2003.

[43] A. F. Molisch, K. Balakrishnan, C. C. Chong, et al., "IEEE 802.15.4a channel model-final report," September 2004, http://www.ieee802.org/15/pub/TG4a.html.

[44] M. Abramowitz and I. A. Stegun, Eds., Handbook of Mathematical Functions with Formulas, Graphs, and Mathematical Tables, Dover, New York, NY, USA, 1972.

[45] M. Basseville and I. V. Nikiforov, Detection of Abrupt Changes: Theory and Application, Prentice-Hall, Englewood Cliffs, NJ, USA, 1993.

[46] H. V. Poor, An Introduction to Signal Detection and Estimation, Springer, New York, NY, USA, 2nd edition, 1994.

[47] P. A. Humblet and M. Azizoğlu, "On the bit error rate of lightwave systems with optical amplifiers," Journal of Lightwave Technology, vol. 9, no. 11, pp. 1576-1582, 1991.

[48] J. G. Proakis, Digital Communication, McGraw-Hill, New York, NY, USA, 4th edition, 2001.

[49] A. F. Molisch, D. Cassioli, C. C. Chong, et al., "A comprehensive standardized model for ultrawideband propagation channels," IEEE Transactions on Antennas and Propagation, vol. 54, no. 11, part 1, pp. 3151-3166, 2006. 